Sitios de patrimonio mundial como determinantes de la demanda de turismo internacional en Latinoamérica y Caribe

Viviana Leonardi, Silvina Elías y Marina Tortul 
Lecturas de Economia, 96 (enero-junio 2022), pp. 171-200

Viviana Leonardi, Silvina Elias y Marina Tortul

\section{Sitios de patrimonio mundial como determinantes de la demanda de turismo internacional en Latinoamérica y Caribe}

Resumen: La literatura destaca una relación positiva entre el Patrimonio de la Humanidad y el turismo receptivo, sobre todo internacional. El objetivo del presente articulo es medir el impacto que tiene la cantidad de Sitios de Patrimonio Mundial de los paises de América Latina y el Caribe sobre el arribo de turistas internacionales. Para ello, siguiendo a Suy Lin (2014), se estiman distintas variantes de la función de demanda de turismo internacional mediante un modelo de datos de panel de 32 países de la región latinoamericana para el período 1995-2016. Losprincipales resultados comprueban que tanto los sitios materiales como los inmateriales tienen un efecto positivo en la llegada de turistas internacionales. Además, se encuentra que dicho efecto es mayor en los paises de ingreso medio alto y alto de la región. En este sentido se considera relevante que los paises de América Latina y el Caribe abonden esfuerzos por lograr el reconocimiento de nuevos Sitios de Patrimonio Mundial.

Palabras clave: turismo, sitios del patrimonio mundial, América Latina y el Caribe, demanda turística, datos de panel.

Clasificación JEL: Z30, Z31, C13, A10.

\section{World Heritage Sites as Determinants of International Tourism Demand in Latin America and the Caribbean}

Abstract: The literature highlights that the declaration of a touristic place as a World Heritage Site (WHS) entails a significant increase in visitors, especially international ones. In this context, the aim of this work is to measure this relationship in the case of the countries of Latin America and the Caribbean. To do so, according to Su and Lin (2014), different variants of the international tourism demand function are estimated using a panel data model of 32 countries in the Latin American region for the period 1995-2016. The main result is that both material and immaterial WHS have a positive effect on the arrival of international tourists to the countries of the studied region. We also found that increasing the number of WHS has a stronger impact on high income countries than on low-income ones. In this sense, it is considered relevant that Latin American and Caribbean countries deepen their efforts to achieve the recognition of new WHS.

Keywords: tourism, world heritage site, Latin America and the Caribbean, tourism demand, panel data.

https://doi.org/10.17533/udea.le.n96a342804

\section{(cc) BY-NC-SA}

Este artículo y sus anexos se distribuyen por la revista Lecturas de Economía bajo los términos de la Licencia Creative Commons Atribución-NoComercial-CompartirIgual 4.0. https://creativecommons.org/licenses/by-nc-sa/4.0/ 


\section{Sites du patrimoine mondial déterminants quant à la demande touristique internationale en Amérique latine et dans les Caraïbes}

Résumé: La littérature met en évidence une relation positive entre le site du Patrimoine de l'Humanité et le tourisme récepteur, en particulier international. L'objectif de cet article est de mesurer l'impact du nombre de sites du Patrimoine de l'Humanité dans les pays d'Amérique Latine et des Caraïbes, sur l'arrivée des touristes internationaux. Pour ce faire, à la suite de Su et Lin (2014), différentes variantes de la fonction de la demande touristique internationale sont estimées à l'aide d'un modèle de données d'un panel de 32 pays de la région de l'Amérique latine pour la période 1995-2016. Les principaux résultats prouvent que les sites tangibles et immatériels ont un effetpositif sur l'arrivée des touristes internationaux. En outre, cet effet s'avère plus important dans les pays de la région ayant un revenu moyen et moyensupérieur. En ce sens, il est jugé pertinent que les pays d'Amérique latine et des Caraïbes intensifient leurs efforts pour parvenir à la reconnaissance de nowveaux sites du Patrimoine de l'Humanité.

Mots clés: tourisme, sites du patrimoine mondial, Amérique latine et Caraïbes, demande touristique, données de panel.

Cómo citar / How to cite this item:

Leonardi V., Elías S., \& Tortul M. (2022). Sitios de patrimonio mundial como determinantes de la demanda de turismo internacional en Latinoamérica y Caribe. Lecturas de Economia, 96, 171-200. https://doi.org/10.17533/udea.le.n96a342804 


\title{
Sitios de patrimonio mundial como determinantes de la demanda de turismo internacional en Latinoamérica y Caribe
}

\author{
Viviana Leonardi $\oplus^{\mathrm{a}}$, Silvina Elías $\oplus^{\mathrm{b}}$ y Marina Tortul $\oplus^{\mathrm{c}}$
}

Introducción. -I. Antecedentes. -II. Metodología. -III. Resultados y Discusión.

-Conclusiones. -Agradecimientos. -Referencias.

Primera versión recibida el 11 de julio de 2020; versión final aceptada el 31 de mayo de 2021

\section{Introducción}

La literatura reconoce el impulso que el turismo ejerce sobre el crecimiento y el desarrollo económico de los países receptores (Almirón et al., 2008; Brida, et al., 2008; Brida, Lanzilotta et al, 2011; Brida et al., 2013; Cuadrado y López, 2015; Rodríguez, 2015). Dicha relación positiva se fundamenta en que el turismo genera directa e indirectamente un aumento de la actividad económica en los lugares visitados, e incluso más allá de estos, debido a la demanda adicional de bienes y servicios que generan los turistas. Así, el turismo impacta sobre el empleo, el producto y, si se considera el turismo internacional, también sobre la balanza de pagos.

Sin embargo, la gran afluencia de turistas, en contraposición a los mencionados beneficios económicos, puede generar conflictos para los países receptores (Ashworth \& Van Der Aa, 2002). Frente a esta problemática, las

a Viviana Leonardi: profesora adjunta e investigadora de la Universidad Nacional del Sur (UNS), Instituto de Investigaciones Económicas y Sociales del Sur (UNS-CONICET), Departamento de Economía, Bahía Blanca, Argentina. Dirección electrónica: vleonard@criba.edu.ar https:// orcid.org/0000-0002-4289-5039

b Silvina Elías: profesora asociada e investigadora de la Universidad Nacional del Sur (UNS), Instituto de Investigaciones Económicas y Sociales del Sur (UNS-CONICET), Departamento de Economía, Bahía Blanca, Argentina. Dirección electrónica: selias@uns.edu.ar https:// orcid.org/0000-0001-7750-1821

c Marina Tortul: ayudante de docencia A y profesional adjunta de la Universidad Nacional del Sur (UNS), Instituto de Investigaciones Económicas y Sociales del Sur (UNS-CONICET), Departamento de Economía, Bahía Blanca, Argentina. Dirección electrónica: mtortul@iiess-conicet.gob.ar https:/ / orcid.org/0000-0002-8739-6049 
Leonardi, Elías y Tortul: Sitios de patrimonio mundial como determinantes de la demanda...

Naciones Unidas consideran que los sitios culturales y paisajes naturales de "valor universal excepcional" deben ser objeto de cuidado y preservación. Así pues, con el objetivo principal de proteger, mantener, entender y poner en valor cultural a dichos sitios, la Organización de las Naciones Unidas para la Educación, la Ciencia y la Cultura (UNESCO) los declara patrimonio de la humanidad o Sitios de Patrimonio Mundial (SPM).

Específicamente, mediante la Convención del Patrimonio Mundial de 1972, la UNESCO elabora la lista de sitios de patrimonio mundial donde se detallan distintas áreas geográficas con características naturales o culturales excepcionales que deben ser cuidadas y preservadas. Esta declaración constituye una herramienta para afrontar los desafíos contemporáneos relacionados con el turismo de masas, con el cambio climático, la urbanización descontrolada, el desarrollo socioeconómico sostenible y las catástrofes naturales (UNESCO, s. f.). Las directrices y criterios para que un sitio obtenga una declaratoria de SPM se encuentra en los textos básicos de la Convención de Patrimonio Mundial de 1972, donde también se detalla el procedimiento para su inscripción ${ }^{1}$. Asimismo, los SPM pueden ser inscritos en la Lista del Patrimonio Mundial en Peligro cuando se considere que están amenazados por peligros comprobados o potenciales, como el deterioro debido a una urbanización galopante o a la explotación no sostenible.

UNESCO (s. f.) diferencia entre sitios del patrimonio material — tangiblese inmaterial —intangibles_- Los primeros se refieren a espacios concretos con relevancia cultural o natural. Los segundos, a distintas manifestaciones de cultura como: tradiciones y expresiones orales, incluido el idioma; artes del espectáculo; usos sociales, rituales y actos festivos; conocimientos y usos relacionados con la naturaleza y el universo; técnicas ancestrales tradicionales. Asimismo, se incluyen los instrumentos, objetos, artefactos y espacios culturales que son inherentes a las prácticas y expresiones culturales. En otras palabras, el patrimonio cultural inmaterial incluye prácticas y expresiones vivas heredadas de los antepasados y transmitidas a los descendientes.

1 Estos lineamientos pueden leerse en Comité Intergubernamental de protección del Patrimonio Mundial cultural y natural (2005). 
Existen dos tipos de listas de patrimonio cultural inmaterial. Por un lado, la Lista de Patrimonio Cultural Inmaterial que requiere medidas urgentes de salvaguardia para asegurar su trasmisión, que se crea en 2003 mediante la Convención para la Salvaguardia del Patrimonio Cultural Inmaterial. Estas inscripciones contribuyen a movilizar la cooperación y la asistencia internacional para que los actores interesados puedan tomar medidas de salvaguardia adecuadas. Por otro lado, se encuentra la lista representativa del Patrimonio Cultural Inmaterial de la Humanidad, que está conformada por expresiones que ilustran la diversidad del patrimonio inmaterial y contribuyen a una mayor conciencia de su importancia. Adicionalmente, existe el Registro de Buenas Prácticas de Salvaguardia. Este registro se compone de programas, proyectos y actividades que mejor reflejen los principios y objetivos de la convención y tiene por objetivo desarrollar medidas de salvaguardia para garantizar la viabilidad de las expresiones orales, la música y los saberes vinculados al arte textil y las tecnologías agrícolas.

Finalmente, existe la Lista Indicativa. La misma es la lista de elementos que un Estado parte de la Convención de 1972 tiene la intención de proponer para su inclusión en la Lista del Patrimonio Mundial en el transcurso de los próximos cinco o diez años.

Adie y Hall (2016) denominan al turismo vinculado a SPM turismo de patrimonio de la humanidad. En muchos casos, el hecho de pertenecer a la Lista de Sitios de Patrimonio Mundial implica un incremento significativo de los visitantes, sobre todo internacionales (Breakey, 2012; Tucker \& Emge, 2010; Tucker \& Carnegie, 2014). No obstante, cada uno de los destinos muestra realidades diversas, pudiendo distinguir claramente entre los territorios consolidados turísticamente y los lugares emergentes. En los primeros, como la Catedral de Notre Dame en París, la Sagrada Familia en Barcelona (Palau-Saumell et al., 2013), las Montañas Azules de Australia (Hardiman \& Burgin, 2013), entre otros, esta relación no es tan clara, pues turísticamente ya atraían a miles de visitantes desde antes de ser SPM. Incluso la literatura muestra que, en algunos de estos casos, la inscripción de un sitio en la lista no coincide siempre con un aumento de las tasas de visita (Buckley, 2004; Patuelli et al., 2013). 
Leonardi, Elías y Tortul: Sitios de patrimonio mundial como determinantes de la demanda...

En particular, es de esperar que el turismo de patrimonio de la humanidad sea cada vez más relevante en la región de América Latina y el Caribe (ALC). Esto se basa en que, por un lado, la región se consolida cada vez como destino turístico internacional. El Barómetro del Turismo Mundial de la Organización Mundial del Turismo (OMT, 2019) da cuenta de ello. Específicamente señala que América del Sur presenta un crecimiento del 7\% entre 2017 y 2018 y Centroamérica y Caribe del $4 \%$. Además, según las previsiones del mismo organismo, esta tendencia continuará hasta el año 2030. Por otro lado, los países de la región inscriben un número creciente de sitios en la lista. De acuerdo con Leonardi et al. (2018), ALC pasa de tener 66 declaraciones en 1995 a 192 en 2016. Este crecimiento se acelera en 2008, cuando se comienzan a incluir los sitios inmateriales — del total de declaraciones existentes en 2016, el $29 \%$ corresponde a la clase intangible-.

No obstante, si bien existen valiosos trabajos empíricos — por ejemplo Galvis y Aguilera (1999) — , aún queda camino por recorrer en el estudio de la relación entre el turismo y el patrimonio de la humanidad en los países de ALC. Al respecto, Ruiz y Pulido (2015) explican que gran parte de las investigaciones científicas publicadas en la base de datos Scopus sobre estos temas, se refieren principalmente a países como Estados Unidos, Reino Unido, China, Australia e Italia. Entre la literatura latinoamericana se pueden mencionar los aportes de Porto et al. (2017), que utilizan modelos gravitacionales basados en datos de panel para estimar la demanda turística en función de la accesibilidad y la dotación de SPM de los países.

En este contexto, el objetivo general de este artículo es aportar evidencia empírica al respecto de la relación entre los SPM y la demanda de turismo internacional en ALC. Se plantean dos objetivos específicos. Primero, medir el impacto de la cantidad de SPM sobre la llegada de turistas internacionales a los países de ALC. Segundo, detectar diferencias estructurales de dicho efecto según el nivel de ingreso de los mencionados países destino. Como caso particular de este último objetivo, se propone identificar el efecto cuantitativo que podría tener la declaración como SPM de todos los elementos que componen la Lista Indicativa. Para ello, se estimarán distintas variantes de la función de demanda de turismo internacional para el período 1995-2016 mediante un modelo de datos de panel. 
El resto del trabajo se organiza como sigue. En la sección I, se revisan algunos antecedentes del efecto del ingreso a la Lista de Sitios de Patrimonio Mundial. En la sección II se explicita la metodología utilizada para alcanzar los objetivos del trabajo y se describe la base de datos. En la sección III se presentan y discuten los resultados de las estimaciones. Finalmente, se concluye.

\section{Antecedentes}

Los bienes SPM se convierten en un punto de atracción turística del mundo. La creciente curiosidad por conocer las distintas culturas se plasma en un número cada vez mayor de visitantes a estos sitios, lo que también impacta en la economía del país. Así, se presentan nuevas oportunidades de empleo, mejora la calidad urbana, la vida social y aumenta aún más el número de turistas. A su vez, esta afluencia de turistas y el reconocimiento que obtienen los SPM les facilita el proceso de conseguir patrocinadores para su mantenimiento y conservación.

En la literatura hay numerosos estudios que intentan estimar tanto el impacto económico real de la inscripción en la Lista de Sitios del Patrimonio Mundial, como el ocurrido sobre la llegada de turistas. Las primeras investigaciones al respecto indican que - efectivamente- una mayor cantidad de SPM acrecienta el turismo (Ashworth \& Turnbridge, 2000; Drost, 1996; Herbert, 2001; Pocock, 1997; Shackley, 1998).

Sin embargo, los estudios empíricos realizados a partir del año 2000 arrojan resultados diversos. Algunos muestran que el nexo entre SPM y la cantidad de visitantes es débil para los sitios que fueron importantes puntos de interés antes de ingresar en la lista. Sitios que son conocidos a nivel mundial como las pirámides de Egipto, el Taj Mahal, etcétera, parecen beneficiarse menos de la marca Patrimonio Mundial en relación con los sitios que poseen un perfil global más bajo. Así, en SPM no tan conocidos a nivel mundial, como Safranbolu, en Turquía, la sociedad receptora ha observado en un período de cinco años un cambio importante, traducido en mejor empleo, oportunidades de negocio y desarrollo comunitario (Türker, 2013). 
Leonardi, Elías y Tortul: Sitios de patrimonio mundial como determinantes de la demanda...

En países como Italia, Patuelli et al. (2013) encontraron que el reconocimiento de la UNESCO no influye en las llegadas de los visitantes, pero sí podría tener un efecto en la competencia mundial, ya que estos sitios tienen la potencia suficiente para motivar el desplazamiento y disfrute de los mismos. Sin embargo, sí se destaca en la literatura que la situación de ser SPM conduce a un aumento en el número de visitantes internacionales, quienes tienden a permanecer más tiempo y a gastar más que los visitantes nacionales (Su \& Lin, 2014; Tucker \& Emge, 2010; Van Der Aa, 2010;). Para Nguyen y Cheung (2014), la inscripción como SPM supone - en la mayoría de los casos- un incremento significativo del número de visitantes a esa zona geográfica, y — por lo tantoun aumento de los ingresos económicos derivados del turismo. También hay estudios que discuten el alcance real de esta relación y la asocian con factores que caracterizan a los diferentes contextos y a la ausencia o insuficiencia de datos en la investigación (Buckley, 2004; Tisdell \& Wilson, 2001).

Otros autores plantean la dualidad entre los beneficios y los costos de los SPM. La consultora Pricewaterhouse Coopers (2007) realiza un amplio análisis para los SPM del Reino Unido. El informe plantea que, pese —a que los beneficios son más que tangibles, especialmente en visibilidad, posicionamiento y número de turistas - hay múltiples costos como la promoción, los estudios técnicos, la gestión del monumento recién designado, los costos de oportunidad — desarrollos urbanísticos posteriores - o, quizá, una masificación del turismo en la zona. Por su parte, Kayahan y Vanblarcom (2012) comparan dos monumentos canadienses y concluyen que, si bien el crecimiento de turistas y de ingresos es positivo en ambos, sólo en el más grande de los dos, los beneficios superan ampliamente a los costos.

Porto et al. (2017) realizan trabajos empíricos para 17 países de América Latina, donde utilizan modelos gravitacionales basados en datos de panel para estimar la demanda turística en función de la accesibilidad y la dotación de SPM de los países. Encuentran que la declaración de atractivos turísticos como sitios patrimoniales por la UNESCO, así como el componente de accesibilidad, incrementan la demanda turística internacional.

A partir de esta revisión, se puede establecer que la literatura no es concluyente respecto al impacto positivo que tiene para un país la inclusión 
de su patrimonio en la lista de la UNESCO, por lo que este artículo intenta hacer un aporte en ese sentido para la región de ALC.

\section{Metodología}

Para abordar el primer objetivo específico, estudiar el efecto de los SPM sobre la llegada de turistas internacionales a los países de ALC, siguiendo a Su y Lin (2014), se estima la función de demanda turística internacional. El modelo general a estimar es (ecuación 1):

$$
y_{i t}=a+b x_{i t}+c z_{i t}+q_{i}+e_{i t},
$$

donde $y_{i t}$ es la llegada de turistas internacionales. Los subíndices $i$ y $t$ denotan el país de destino y el período de tiempo, respectivamente. El término $x_{i t}$ incluye las principales variables explicativas; $z_{i t}$, las variables de control que también afectan a la demanda y que normalmente se utilizan en la literatura. $q_{i}+e_{i t}$, el error compuesto de la estimación, $q_{i}$ captura los efectos individuales y $e_{i t}$, el error estocástico con las propiedades tradicionales. $a$ es la constante de la estimación. $b$ y $c$ son los vectores de coeficientes de las variables explicativas y de control, respectivamente.

Para estimar este modelo se considera como proxy de la demanda turística internacional, la llegada de turistas internacionales al país $i$ en el momento $t$ - $_{\text {ATUR }}{ }_{i t}$ - (Lim, 2006; Song et al., 2010). Como principal variable explicativa $\left(x_{i t}\right)$, se define la cantidad de SPM total que tiene cada país en cada año del período $\left(\mathrm{SPMT}_{i t}\right)$. Luego, se consideran separadamente la cantidad de SPM materiales (SPMM) e inmateriales (SMPI). Esta distinción se basa en el comportamiento diferencial del cada tipo de $\mathrm{SPM}^{2}$. En particular, se observa la creciente importancia relativa de los últimos, que pasan de representar el

2 El comportamiento diferencial se fundamenta no solo en la naturaleza del patrimonio tangible e intangible, sino en los tiempos en que se consideró la necesidad de su protección. Así, si bien el patrimonio cultural, tanto material como inmaterial, sienta las bases para el desarrollo del turismo cultural, la necesidad de protección del patrimonio intangible surge con posterioridad a la idea de proteger conjuntamente los sitios naturales y los culturales, demorándose aquellas medidas destinadas a garantizar su viabilidad. Esta demora se traduce en la necesidad de profundizar el conocimiento y la reflexión acerca de las políticas patrimoniales más adecuadas para su promoción. 
Leonardi, Elías y Tortul: Sitios de patrimonio mundial como determinantes de la demanda...

$15 \%$ en 2008, al $29 \%$ en 2016. Se espera que el signo de los coeficientes de estas variables sea positivo, indicando que el aumento de SPM incrementa la llegada de turistas internacionales. En cuando al efecto diferencial de los SPMM y SPMI, se espera que el de los primeros supere al de los segundos, en tanto que los materiales datan de mayor tiempo en Lista $^{3}$ y por lo tanto gozan de mayor reconocimiento mundial.

Entre las variables de control $\left(z_{i t}\right)$, se consideran:

- El producto interno bruto per cápita $\left(\mathrm{PBIpc}_{i t}\right)$, como proxy del grado de desarrollo económico del país de destino ${ }^{4}$. Se mide en términos de paridad del poder adquisitivo en dólares constantes de 2011. Se espera un coeficiente positivo, indicando que los turistas internacionales prefieren viajar a países más desarrollados.

- El tipo de cambio oficial (TCE $i t$ ) definido como unidades de moneda local por unidad de dólar. Se espera un coeficiente positivo, pues si TCE it $_{\text {it }}$ aumenta, se produce una depreciación de la moneda local y disminuye el costo de viajar al país de destino.

- El gasto en salud como porcentaje del PBI (Producto Bruto Interno) $\left(\mathrm{SALU}_{i t}\right)$, como proxy de la condición sanitaria del país de destino. Se espera un coeficiente positivo ya que, si un país gasta menos en el cuidado de la salud de sus residentes, tiende a empeorar la condición sanitaria del país en general, esto podría aumentar el riesgo de contraer enfermedades para los turistas internacionales y por lo tanto podría desalentar su ingreso ${ }^{5}$.

3 Los primeros SPM declarados en ALC fueron de tipo material en 1978, mientras que recién en el año 2008 se declaran en varios países de la región diferentes prácticas y elementos de tipo inmaterial.

4 Su y Lin (2014) y Roh et al. (2015) consideran como variables de control el PBI y la población.

5 Otras posibles variables explicativas como educación - medida por la tasa de escolarización de nivel terciario de acuerdo con Roh et al., (2015)— y transparencia — que combina un índice de transparencia y corrupción — no se tuvieron en cuenta en las estimaciones dada la disponibilidad de datos y la correlación significativa con PBIpc. 
Es válido aclarar que las características diferenciales del país de origen también pueden influir en la demanda turística. Sin embargo, dado que los datos discriminados por lugar de origen no están disponibles para todos los países de la muestra, los efectos del nivel de ingreso y población de los países emisores no están considerados.

De esta forma se especifican dos funciones generales de demanda turística internacional. En la primera (ecuación 2) se considera el total de SPM de cada país y en la segunda (ecuación 3), a los fines de comprender la influencia de las distintas clases de SPM sobre el arribo de turistas, se realiza la distinción entre sitios materiales e inmateriales.

$$
\begin{gathered}
A T U R_{i t}=a+b_{1} S P M T_{i t}+c_{1} P B I p c_{i t}+c_{2} T C E_{i t}+c_{3} S A L U_{i t}+q_{i}+e_{i t} \\
\begin{array}{r}
A T U R_{i t}=a+b_{2} S P M M_{i t}+b_{3} S P M I_{i t}+c_{1} P B I p c_{i t} \\
+c_{2} T C E_{i t}+c_{3} S A L U_{i t}+q_{i}+e_{i t} .
\end{array}
\end{gathered}
$$

Para abordar el segundo objetivo específico, detectar diferencias estructurales en las funciones de demanda de turismo internacional según el nivel de ingreso de los países destino, se clasifica la muestra en países de ingreso alto y medio-alto y en países de ingreso medio bajo y bajo, de acuerdo con la clasificación del Banco Mundial (s. f. ${ }^{6}$. Entre los primeros se incluyen: Antigua y Barbuda, Argentina, Bahamas, Barbados, Belice, Brasil, Chile, Colombia, Costa Rica, Dominica, República Dominicana, Ecuador, Granada, Guyana, Jamaica, México, Panamá, Paraguay, Perú, San Kitts y Nevis, Santa Lucía, San Vicente y las Granadinas, Surinam, Trinidad y Tobago, Uruguay y Venezuela. Y entre los segundos: Bolivia, El Salvador, Guatemala, Haití, Honduras y Nicaragua. Esta clasificación desbalancea la base de datos en tanto que el grupo de países de menores ingresos es sustancialmente más reducido, lo cual podría afectar a la robustez de los resultados.

Para estimar las funciones de demanda, primero se corrobora la presencia de heterogeneidad de corte horizontal mediante los test $\mathrm{F}$ de restricciones

6 De acuerdo con la clasificación hist órica del Banco Mundial (s. f.), algunos países particulares cambian de categoría de ingresos durante el período 1995-2016. Se considera la clasificación de 2016 ya que en general representa la mayor continuidad de cada país en la misma categoría. 
Leonardi, Elías y Tortul: Sitios de patrimonio mundial como determinantes de la demanda...

- de que todos los efectos individuales son nulos-y de Breusch-Pagan (BP). Se realizan las estimaciones mediante un modelo de datos de panel, ya que mínimos cuadrados ordinarios agrupados generaría estimadores inconsistentes. Para elegir entre modelos de datos de panel de efectos fijos o variables, se utiliza la prueba de Hausman. Los modelos de efectos aleatorios indican que las características individuales no observables no están correlacionadas con las variables explicativas, lo cual es poco probable cuando la unidad observacional son países y las explicativas son variables macroeconómicas. Para evaluar la bondad de ajuste se presenta el $\mathrm{R}^{2}$ overall. En todos los casos se considera un error de $5 \%$ y $1 \%$. El análisis se realiza con el software STATA 14.

Finalmente, el efecto de incorporar todos los sitios de la Lista Indicativa a la Lista de Sitios de Patrimonio Mundial sobre la llegada de turistas internacionales se calcula según la ecuación 4.

$$
\triangle A T U R_{i}=b_{1} Q L I_{i}
$$

donde: $A T U R_{i}$ representa el arribo de turistas internacionales al país $i . b_{1}$ es el coeficiente de SMPT que representa el efecto marginal de un nuevo SPM sobre el arribo de turistas internacionales (ecuación 2). $Q L I_{i}$ es la cantidad de sitios que integran la Lista Indicativa del país $i$.

\section{A. Descripción de la base de datos}

La base de datos está conformada por 32 países de ALC para el período 1995-2016 ${ }^{7}$. Se elabora a partir de la base de datos Indicadores de Desarrollo Mundial del Banco Mundial (s. f. a) y de la Lista de Patrimonio Mundial de la UNESCO (s. f. a) — ver Tabla 1-.

Se trata de un panel de datos desbalanceado (Tabla 2). Con 704 datos genuinos por variable, SPMT, SPMM, SPMI y TCE están balanceadas, no así el resto. En particular, hay 3 datos perdidos de ATUR (Haití, Honduras y St.

De los 42 países que conforman la región ALC — de acuerdo con el Banco Mundial— se excluyen Aruba, Cuba, Curazao, Islas Caimán, Islas Turcas y Caicos, Islas Vírgenes (Estados Unidos), Islas Vírgenes (Gran Bretaña), Puerto Rico, St. Martin (Francia) y St. Martin (Holanda) debido a la disponibilidad de datos relativa a las variables de control. 
Kitts y Navis, todos para 2016); 3 de PBIpc (Haití, 1995 y Venezuela, 1995 y 2016) y 192 de SALU (solo se disponen datos para el período 2000-2015).

Tabla 1. Fuentes de datos

\begin{tabular}{|c|c|c|c|}
\hline Variable & Fuente & Denominación & Fuente primaria \\
\hline ATUR & $\begin{array}{l}\text { Indicadores de De- } \\
\text { sarrollo Mundial, } \\
\text { Banco Mundial }\end{array}$ & $\begin{array}{l}\text { Turismo internacional, } \\
\text { número de arribos }\end{array}$ & $\begin{array}{l}\text { Organización Mundial del Tu- } \\
\text { rismo, Anuario de estadísticas } \\
\text { de turismo, Compendio de es- } \\
\text { tadísticas de turismo y archi- } \\
\text { vos de datos }\end{array}$ \\
\hline PBIpc & $\begin{array}{l}\text { Indicadores de De- } \\
\text { sarrollo Mundial, } \\
\text { Banco Mundial }\end{array}$ & $\begin{array}{l}\text { PIB per cápita, PPA } \\
\text { (precios internacionales } \\
\text { constantes de 2011) }\end{array}$ & $\begin{array}{l}\text { Banco Mundial, base de da- } \\
\text { tos del Programa de Compara- } \\
\text { ción Internacional. }\end{array}$ \\
\hline TCE & $\begin{array}{l}\text { Indicadores de De- } \\
\text { sarrollo Mundial, } \\
\text { Banco Mundial }\end{array}$ & $\begin{array}{l}\text { Tasa de cambio oficial } \\
\text { (UMN por USD, pro- } \\
\text { medio para un período) }\end{array}$ & $\begin{array}{l}\text { Fondo Monetario Internacio- } \\
\text { nal, Estadísticas Financieras } \\
\text { Internacionales. }\end{array}$ \\
\hline SALU & $\begin{array}{l}\text { Indicadores de De- } \\
\text { sarrollo Mundial, } \\
\text { Banco Mundial }\end{array}$ & $\begin{array}{l}\text { Gasto corriente en salud } \\
\text { ( } \% \text { de } \mathrm{PBI})\end{array}$ & $\begin{array}{l}\text { World Health Organization } \\
\text { Global Health Expenditure. }\end{array}$ \\
\hline SPMT & & & $\begin{array}{l}\text { UNESCO, World Heritage } \\
\text { List }\end{array}$ \\
\hline SPMM & & & $\begin{array}{l}\text { UNESCO, World Heritage } \\
\text { List }\end{array}$ \\
\hline SPMI & & & $\begin{array}{l}\text { UNESCO, World Heritage } \\
\text { List }\end{array}$ \\
\hline
\end{tabular}

Fuente: elaboración propia a partir de Banco Mundial (s. f. a) y UNESCO (s. f. a).

Dicha composición de la base de datos acota el período de estimación a los datos disponibles. Para comprobar la robustez de los coeficientes se realizan dos tipos de estimaciones: a) considerando únicamente las variables explicativas principales y b) tanto las principales como las de control. De este modo, las variantes de la función de demanda a estimar son: 
Leonardi, Elías y Tortul: Sitios de patrimonio mundial como determinantes de la demanda...

$$
\begin{aligned}
A T U R_{i t}= & a+b_{1} S P M T_{i t}+q_{i}+e_{i t} \\
A T U R_{i t}= & a+b_{1} S P M T_{i t}+c_{1} P B I p c_{i t}+c_{2} T C E_{i t}+c_{3} S A L U_{i t} \\
& +q_{i}+e_{i t} \\
A T U R_{i t}= & a+b_{2} S P M M_{i t}+b_{3} S P M I_{i t}+q_{i}+e_{i t} \\
A T U R_{i t}= & a+b_{2} S P M M_{i t}+b_{3} S P M I_{i t}+c_{1} P B I p c_{i t} \\
& +c_{2} T C E_{i t}+c_{3} S A L U_{i t}+q_{i}+e_{i t}
\end{aligned}
$$

Se observa una relación directa entre los SPM y ATUR (Tabla 3 y Figura 1), excepto para Venezuela. Posiblemente esto podría estar asociado a su propio escenario sociopolítico.

Tabla 2. Estadística descriptiva. América Latina y El Caribe. 1995-2016

\begin{tabular}{cccccc}
\hline Variable & Observaciones & Media & Desvío Estándar & Mínimo & Máximo \\
\hline ATUR & 701 & 1812995 & 4049806 & 43000 & 35079000 \\
SPMT & 704 & 3,8 & 6,1 & 0 & 42 \\
SPMM & 704 & 3,3 & 5,3 & 0 & 34 \\
SPMI & 704 & 0,5 & 1,3 & 0 & 9 \\
PBIpc & 701 & 11485 & 6075 & 1502 & 31951 \\
TCE & 704 & 384 & 1012 & 0,18 & 11787 \\
SALU & 512 & 6 & 1,4 & 3,1 & 10,6 \\
\hline
\end{tabular}

Fuente: elaboración propia a partir de Banco Mundial (s. f. a) y UNESCO (s. f. a).

Finalmente, la Figura 2 ordena la muestra según la cantidad promedio $\mathrm{SPM}^{8}$. Trinidad y Tobago, San Vicente y Las Granadinas, Las Bahamas y Granada no poseen SPM en el período de estudio, además algunos países no poseen SPM en algunos años. Estos ceros representan valores genuinos y por

8 SPMM también incluyen aquellos inscriptos en la Lista del Patrimonio Mundial en Peligro. SPMI incluyen tanto aquellos inscriptos en la Lista de Patrimonio Cultural Inmaterial como aquellos que requieren medidas urgentes de salvaguardia. 
lo tanto aumentan la variabilidad de las variables, lo cual incide positivamente en la significatividad de sus coeficientes, aunque al tratarse de valores nulos podrían afectar negativamente su magnitud. Sin embargo, se espera que no influyan en los resultados de las estimaciones ya que representan menos del $25 \%$ de las observaciones (173 de 704 observaciones).

Tabla 3. Sitios de Patrimonio mundial y arribo de turistas internacionales. Principales paises de América Latina y El Caribe. 1995 y 2016

\begin{tabular}{|c|c|c|c|c|c|c|c|}
\hline \multirow{2}{*}{ Países } & \multirow{2}{*}{ Años } & \multicolumn{4}{|c|}{ Sitios de Patrimonio Mundial } & \multicolumn{2}{|c|}{ Arribo de turistas internacionales } \\
\hline & & SPMM & SPMI & SPMT & Var. \% & ATUR & Var. \% \\
\hline \multirow{2}{*}{ México } & 1995 & 14 & 0 & 14 & & 20241000 & \\
\hline & 2016 & 34 & 8 & 42 & 200 & 35079000 & 73 \\
\hline \multirow{2}{*}{ Brasil } & 1995 & 8 & 0 & 8 & & 1991000 & \\
\hline & 2016 & 20 & 6 & 26 & 225 & 6578000 & 230 \\
\hline \multirow{2}{*}{ Perú } & 1995 & 9 & 0 & 9 & & 479000 & \\
\hline & 2016 & 12 & 10 & 22 & 144 & 3744000 & 682 \\
\hline \multirow{2}{*}{ Colombia } & 1995 & 5 & 0 & 5 & & 1399000 & \\
\hline & 2016 & 8 & 9 & 17 & 240 & 3317000 & 137 \\
\hline \multirow{2}{*}{ Bolivia } & 1995 & 3 & 0 & 3 & & 284000 & \\
\hline & 2016 & 7 & 4 & 11 & 267 & 959000 & 238 \\
\hline \multirow[t]{2}{*}{ Argentina } & 1995 & 3 & 0 & 3 & & 2289000 & \\
\hline & 2016 & 10 & 2 & 12 & 300 & 5559000 & 143 \\
\hline \multirow{2}{*}{ Venezuela } & 1995 & 2 & 0 & 2 & & 700000 & \\
\hline & 2016 & 3 & 5 & 8 & 300 & 601000 & -14 \\
\hline \multirow{2}{*}{ Ecuador } & 1995 & 3 & 0 & 3 & & 440000 & \\
\hline & 2016 & 5 & 3 & 8 & 167 & 1418000 & 222 \\
\hline \multirow{2}{*}{ Chile } & 1995 & 1 & 0 & 1 & & 1540000 & \\
\hline & 2016 & 6 & 1 & 7 & 600 & 5641000 & 266 \\
\hline \multirow{2}{*}{ Guatemala } & 1995 & 3 & 0 & 3 & & 563000 & \\
\hline & 2016 & 3 & 3 & 6 & 100 & 1906000 & 238 \\
\hline
\end{tabular}

Fuente: elaboración propia a partir de Banco Mundial (s. f. a). 
Leonardi, Elías y Tortul: Sitios de patrimonio mundial como determinantes de la demanda...

Figura 1. Sitios de Patrimonio Mundial y Arribo de Turistas Internacionales. América Latina y El Caribe. 1995 y 2016
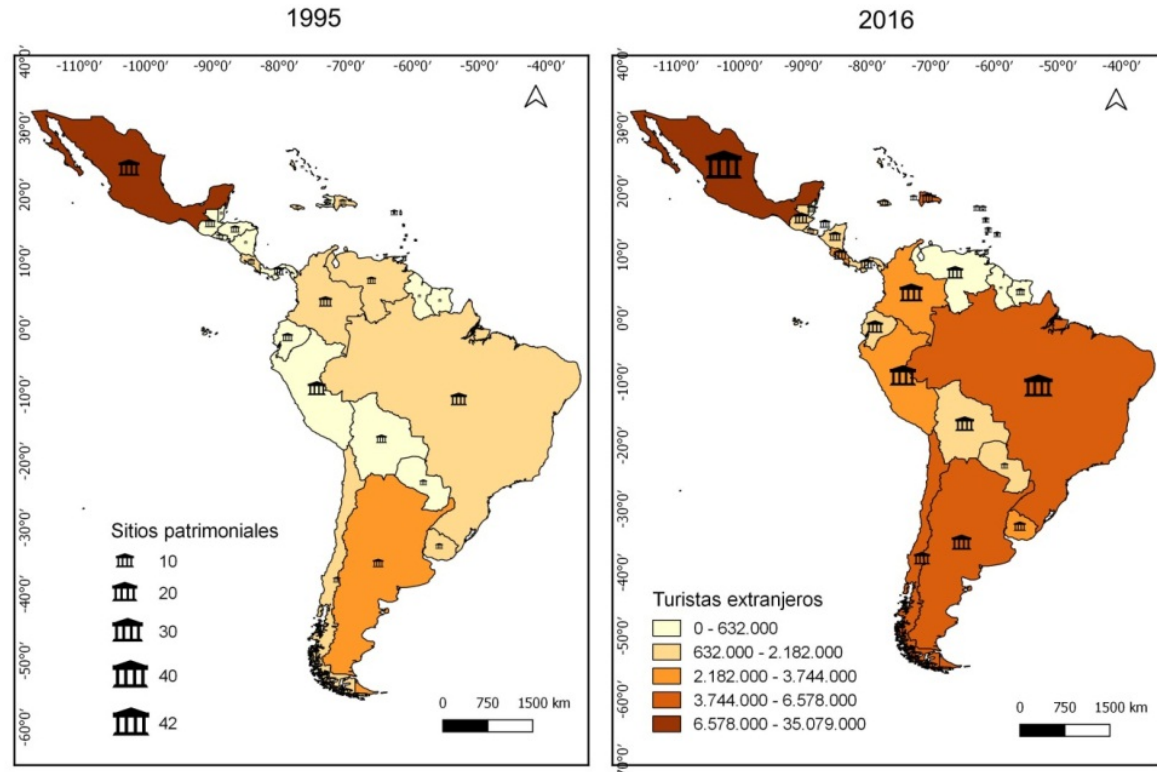

Fuente: elaboración propia en base a la UNESCO (s. f. a) y Banco Mundial (s. f. a).

\section{Resultados y discusión}

\section{A. Estimación de la demanda de turismo internacional: efecto de los SPM sobre la llegada de turistas internacionales a los países de ALC}

La Tabla 4 presenta los resultados de las estimaciones para los 32 países de la muestra. En todos los casos se comprueba la significatividad de los efectos individuales. El modelo de efectos fijos es más apropiado para las variantes 2a, $2 \mathrm{~b}$ y $3 \mathrm{a}$, aunque no para la 3b. En general, los SPM por sí solos explican más del $68 \%$ de la variabilidad de la demanda de turismo internacional de los países del ALC (2a y 3a). Además, el $\mathrm{R}^{2}$ no aumenta sustancialmente cuando se consideran las variables de control ( $2 \mathrm{~b}$ y $3 \mathrm{~b}$, respectivamente), sugiriendo que — en definitiva — la demanda se explica principalmente por la cantidad de SPM. 
Figura 2. Sitios de Patrimonio Mundial. América Latina y El Caribe. Promedio 1995-2016

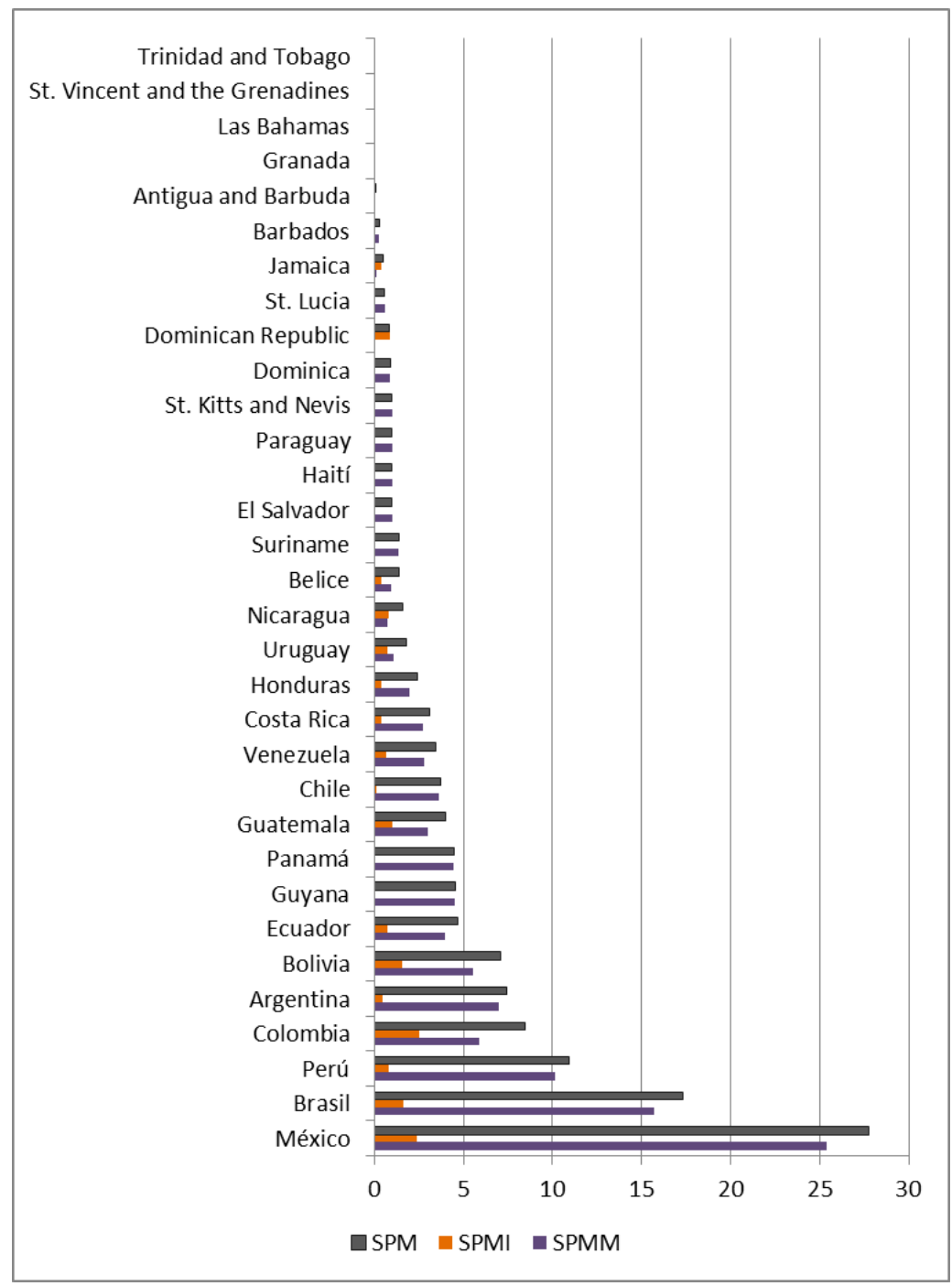

Fuente: elaboración propia a partir de UNESCO (s. f. a). 
Leonardi, Elías y Tortul: Sitios de patrimonio mundial como determinantes de la demanda...

De acuerdo con lo esperado, en todos los casos los coeficientes de SPMT, SPMM y SPMI son significativos y positivos. En cuanto a las variables de control, solo PBIpc es significativa y presenta el signo esperado. Vale destacar que la no significatividad de TCE podría asociase a su poca variabilidad debido a las políticas de tipo de cambio fijo de algunos países. Y la no significatividad de SALU, a que los países podrían ser relativamente homogéneos en términos de su estatus de salud por pertenecer a la misma región. Así, en muestras más amplias y diversas se podrían esperar resultados diferentes (Roh et al., 2015).

Tabla 4. Resultados de la Estimación de la Demanda de Turismo Internacional. América Latina y El Caribe. 1995-2016

\begin{tabular}{lcccc}
\hline \multirow{2}{*}{ Variables independientes } & \multicolumn{4}{c}{ Variable dependiente: ATUR } \\
\cline { 2 - 5 } SPMT & $296801^{* * *}$ & $272894^{* * *}$ & $3 \mathrm{a}$ & $3 \mathbf{b}$ \\
SPMM & & $315019^{* * *}$ & $491344^{* * *}$ \\
SPMI & & $272789^{* * *}$ & $112756^{* * *}$ \\
PBIpc & & $79^{* * *}$ & & $82^{* * *}$ \\
TCE & 109 & & 82 \\
SALU & & 23376 & & 54994 \\
Const. & $671507^{* * *}$ & -362137 & $622600^{* * *}$ & $-1269699^{* * *}$ \\
Hausman & $417^{* * *}$ & $84^{* * *}$ & $22^{* * *}$ & 2 \\
$\mathrm{R}^{2}$ Overall & 0,6812 & 0,707 & 0,6861 & 0,7326 \\
Test F/ BP & $273^{* * *}$ & $275^{* * *}$ & $256^{* * *}$ & $3411^{* * *}$ \\
Obs. & 701 & 511 & 701 & 511 \\
\hline ** p-value $<0,05 ;{ }^{* * *}$ p-value $<0,01$. & & &
\end{tabular}

Fuente: elaboración propia

En cuanto a la magnitud de los coeficientes, adicionar un nuevo SPM a la lista incrementa la demanda de turismo internacional en más de 270 mil turistas al año (2b). Asimismo, como se esperaba, se observa que el impacto de los SPMM es mayor que el de los SPMI (3a y 3b). Además, esta diferencia se acrecienta al considerar las variables de control. Teniendo en cuenta 
que la literatura documenta la relación positiva entre turismo y crecimiento económico, esto coloca a las declaratorias de SPM como una herramienta de desarrollo más a disposición de los países de ALC. Aún más, de acuerdo con estos resultados, los países de ALC deberían concentrarse sobre todo en las nominaciones de los SPMM. Sin embargo, Roh et al. (2015) encuentran un efecto positivo mayor de la cantidad de sitios inmateriales que materiales, y lo atribuyen a las asimetrías de información. Como se mencionará, para la muestra de países estudiada dicha relación no se observa. Esto podría deberse a que, los SPMI datan de menor tiempo en la lista, y por lo tanto, su gestión en ALC aún requiere profundizar el conocimiento y la reflexión acerca de las políticas patrimoniales más adecuadas para su promoción, tal como queda plasmado por la UNESCO (2021). Así, el menor tiempo en la lista de los SPMI, implica un menor reconocimiento mundial de los mismos.

\section{B. Estimación de la demanda de turismo internacional: diferencias estructu- rales según el nivel de ingreso de los países destino de ALC}

La Tabla 5 resume los resultados de las estimaciones de demanda distinguiendo a los países según su nivel de ingreso. Como en el caso general, el modelo de efectos fijos ajusta mejor al grupo de mayores ingresos, mientras que el de efectos aleatorios al de menores (excepto la especificación 3b). A su vez, los resultados en cuanto a la significatividad y signo de las variables explicativas del primer grupo son semejantes a los de la muestra en general, lo cual es de esperar dado que 26 de los 32 países estudiados son de ingreso alto y medio-alto.

En cambio, los resultados difieren para el segundo grupo. Tan es así que, si bien SPMT es significativa y con el signo esperado (2a), la magnitud del coeficiente es menos de la mitad que aquel del otro grupo. Esto indica que la demanda de turismo internacional es más sensible a la cantidad SPM en los países de ALC de mayores ingresos que en la de menores. Aún más, en este último caso, SPMT solo explica el $6 \%$ de la variabilidad de ATUR, mientras que en el primero esta proporción asciende a $70 \%$ (2a). Lo cual sugiere que la cantidad de SPM no es una variable central a la hora de decidir el destino turístico entre los países de menores ingresos de ALC. 
Leonardi, Elías y Tortul: Sitios de patrimonio mundial como determinantes de la demanda...

Tabla 5. Resultados de la estimación de la demanda de turismo internacional según categoría de ingreso del país receptor. América Latina y El Caribe. 1995-2016

\begin{tabular}{|c|c|c|c|c|c|c|c|c|}
\hline \multirow{3}{*}{$\begin{array}{l}\text { Variables inde- } \\
\text { pendientes }\end{array}$} & \multicolumn{8}{|c|}{ Variable dependiente: ATUR } \\
\hline & \multicolumn{2}{|c|}{$2 a$} & \multicolumn{2}{|c|}{$2 \mathrm{~b}$} & \multicolumn{2}{|c|}{$3 a$} & \multicolumn{2}{|c|}{$3 b$} \\
\hline & I.Alto & I.Bajo & I.Alto & I.Bajo & I.Alto & I.Bajo & I.Alto & I.Bajo \\
\hline SPMT & $309800^{* * *}$ & $122639^{* * *}$ & $287620^{* * *}$ & -23953 & & & & \\
\hline SPMM & & & & & $310775^{* * *}$ & -17926 & $489381^{* * *}$ & $-328526^{* * *}$ \\
\hline SPMI & & & & & $308395^{* * *}$ & $186034^{* * *}$ & $123732^{* * *}$ & -17746 \\
\hline PBIpc & & & $76^{* * *}$ & $440^{* * *}$ & & & $79^{* * *}$ & $492^{* * *}$ \\
\hline TCE & & & 124 & $15788^{* * *}$ & & & 87 & $11847^{* * *}$ \\
\hline SALU & & & 36622 & 18811 & & & 62475 & $23358^{*}$ \\
\hline Constante & $797283^{* * *}$ & $382848^{* *}$ & -421388 & $-1553798^{* * *}$ & $794438^{* * *}$ & $655303^{* * *}$ & $-1299695^{* * *}$ & $-1059294^{* * *}$ \\
\hline Test Hausman & $216^{* * *}$ & 4 & $11^{* *}$ & 7 & $19^{* * *}$ & 0,13 & 3 & $22^{* *}$ \\
\hline $\mathrm{R}^{2}$ overall & 0,7014 & 0,0613 & 0,7301 & 0,7443 & 0,7016 & 0,2192 & 0,7458 & 0,5867 \\
\hline Test F/ BP & $270^{* * *}$ & $387^{* * *}$ & $264^{* * *}$ & $212^{* * *}$ & $258^{* * *}$ & $428^{* * *}$ & $2699^{* * *}$ & $31^{* * *}$ \\
\hline Obs. & 571 & 130 & 415 & 96 & 571 & 130 & 415 & 96 \\
\hline
\end{tabular}

${ }^{* *}$ p-value $<0,05 ;{ }^{* * *}$ p-value $<0,01$

Fuente: elaboración propia.

Esta idea, se refuerza cuando se controlan las variables macroeconómicas (2b), pues resulta que SPMT no es significativa, pero sí lo son PBIpc -incluso, con un coeficiente cinco veces mayor que en el caso general-y TCE; ambas con el signo esperado. Esto parece señalar que los turistas que visitan los países de menores ingresos de ALC basan su decisión de viaje, en las condiciones macroeconómicas del país de destino. No obstante, este resultado no necesariamente implica que la demanda de turismo internacional de dichos países sea insensible a la cantidad de SPM que poseen, pues regresiones auxiliares denotan la presencia de multicolinealidad entre SPMT y $\mathrm{PBIpc}^{9}$. Además, cuando se distingue entre tipos de patrimonio, solo SPMI es significativa, con el signo esperado y de magnitud similar al caso general (3a).

9 En los países de menores ingresos, el coeficiente de correlación entre SPMT y PBIpc es significativo de $34 \%$. Además, la estimación auxiliar de SMPT en función de PBIpc mediante datos de panel arroja un coeficiente significativo de la explicativa y un $\mathrm{R}^{2}$ de $12 \%$. En el caso de los países de mayores ingresos, el coeficiente de correlación no es significativo, el de la explicativa lo es, aunque el $\mathrm{R}^{2}$ es de apenas $0,01 \%$. 
Sin embargo, al considerar la situación macroeconómica (3b), nuevamente, se observan los efectos de la multicolinealidad ${ }^{10}$. Ya que las variables de control resultan significativas — con el signo esperado_- aunque en el caso de PBIpc se observa una magnitud sustancialmente mayor al caso general, pero los coeficientes de SPMM y SPMI presentan signo o significatividad contraria a lo esperado. En suma, parece que el desafío para los países de menores ingresos de ALC es aún mayor que para los de mayores ingresos, pues para reactivar la economía mediante el turismo internacional podrían requerir no solo incrementar la cantidad de nominaciones, sino — sobre todo- mejorar su entorno macroeconómico.

Finalmente, Brasil, México, Chile, Colombia y Argentina son los países de ALC que más elementos suscriben a la Lista Indicativa. Excepto por Chile, estos son los países de ALC que ya cuentan con la mayor cantidad de nominaciones. Para ellos, el registro como patrimonio mundial de todos los elementos que integran dicha la lista generaría una afluencia adicional de entre 7 y 3 millones de turistas internacionales aproximadamente (Figura 3) ${ }^{11}$.

\section{Conclusiones}

La relación entre el turismo y la inscripción de un lugar como patrimonio de la humanidad, tanto material como inmaterial, se presenta en la literatura de economía del turismo como un aspecto de interés para estudiar. En muchos casos, la inclusión en dichos listados conlleva un incremento significativo de los visitantes — sobre todo extranjeros—- creándose una fuerte relación entre la cantidad de SPM y el turismo internacional.

10 Los problemas de multicolinealidad se agravan en el modelo $3 \mathrm{~b}$ con respecto al $2 \mathrm{~b}$ para el grupo de países de menores, ingresos dado que la correlación entre SPMM y SPMI es significativa de $50 \%$. En los países de mayores ingresos dicha correlación no es significativa.

11 Si bien, para calcular la contribución a la demanda de turismo internacional que podría generar el registro como SPM de los elementos que integran la Lista Indicativa deberían utilizarse los coeficientes del modelo 2 completo (2b), como el mencionado problema de multicolinealidad de la submuestra de países de bajos ingresos no permite rechazar la hipótesis nula de que el coeficiente de la variable SPMT es diferente a cero, se utilizan aquellos provistos por la variante $2 \mathrm{a}$. 
Leonardi, Elías y Tortul: Sitios de patrimonio mundial como determinantes de la demanda...

Figura 3. Incremento en la demanda de turismo internacional dado el reconocimiento como Patrimonio de la Humanidad de los elementos de la Lista Indicativa. América Latina y El

Caribe. 2016

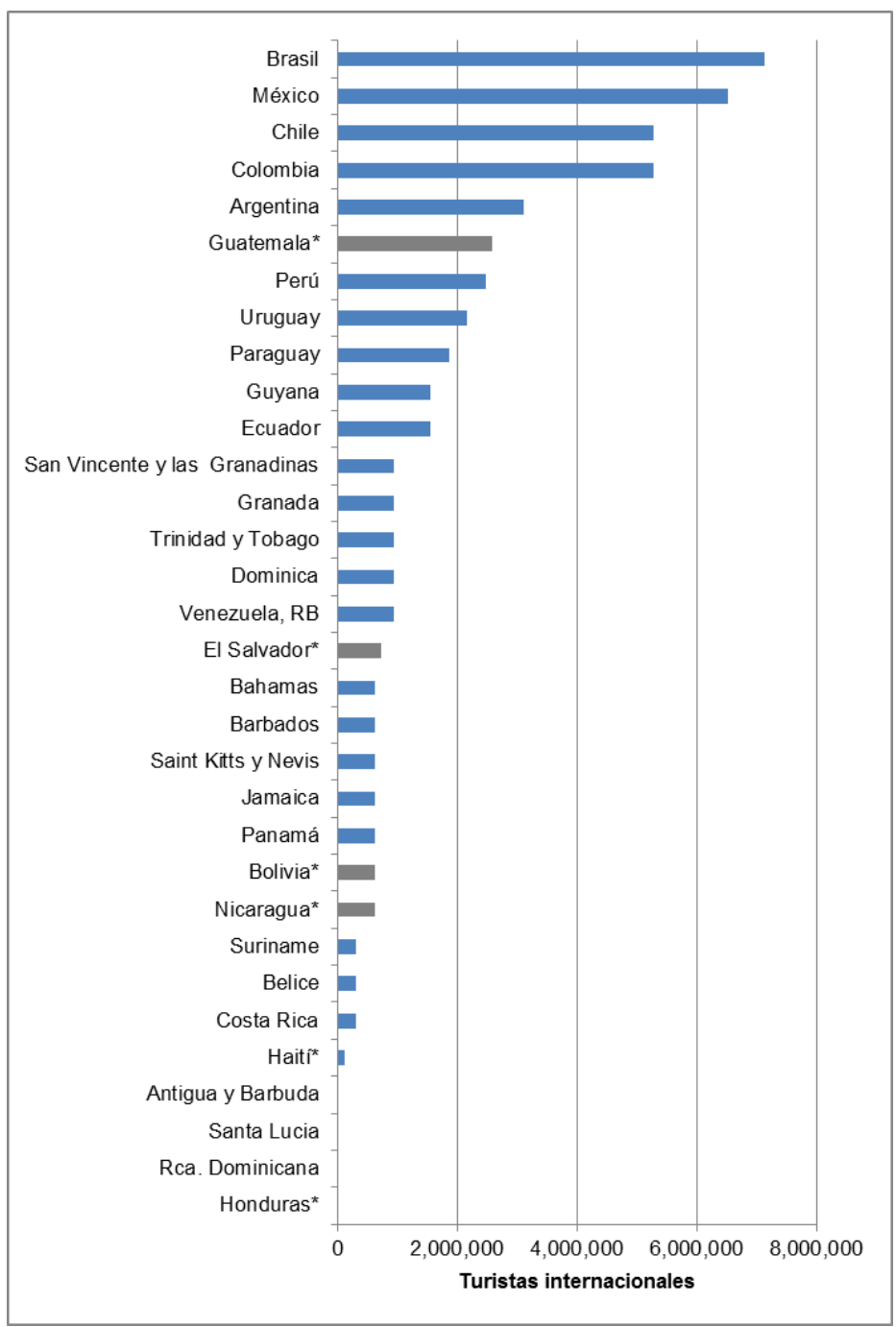

Fuente: elaboración propia. 
En este artículo se estudió el impacto que tiene, sobre la llegada de turistas internacionales a los países de ALC, la cantidad de SPM materiales e inmateriales que poseen estos países. Para ello, se estimaron diferentes variantes de la función de demanda de turismo internacional a partir de un panel de datos de 32 países de la región para el período 1995-2016. Los resultados hallados son parcialmente coincidentes con la literatura precedente (Su \& Lin, 2014; Roh et al., 2015; Porto et al., 2017), en tanto se observa una relación significativamente positiva entre la cantidad de SPM y la afluencia de turistas internacionales hacia los países de ALC. No obstante, al contrario que otros autores, se encuentra que el efecto de los SPMM es mayor que el de los SPMI en el caso de los países analizados.

Además, este artículo avanza en distinguir el impacto que el número de SPM causa sobre el turismo internacional receptivo según el nivel de ingreso de los países destino de ALC. Por un lado, se encuentra que este efecto es significativamente positivo para los países de mayores ingresos. De hecho, el cálculo del aporte potencial al turismo internacional del reconocimiento como patrimonio de la humanidad de los sitios de la Lista Indicativa confirma el planteo: "los procesos de declaratoria patrimonial actúan como legitimadores de atracción convirtiendo a estos elementos, naturales o culturales, tangibles o intangibles, en recursos públicos de alto potencial turístico y fortaleciendo los arribos en los sitios en los que se difunden" (Schenkel \& Pinassi, 2015, p. 50). Sin embargo, para el caso de los países de menores ingresos, la demanda de turismo parece estar más afectada por el nivel de desarrollo del país receptor y por su tipo de cambio que por el número de SPM. Ciertamente este resultado plantea la necesidad de distinguir dos tipos de recomendaciones de política diferentes. Por un lado $-\mathrm{y}$ teniendo en cuenta la relación positiva entre turismo y crecimiento, o aún más desarrollo-, económico que documenta la literatura, se alienta a los países de ingresos altos y medio altos de la región a ahondar esfuerzos en lograr el reconocimiento de nuevos SPM. Pero, no es posible realizar la misma recomendación para los países de ingresos medio bajo y bajos de ALC, pues la evidencia empírica no es contundente en este sentido. Así, podría decirse que — para estos países de menores ingresos - reactivar la economía mediante el turismo internacional podría requerir principalmente la mejora del entorno macroeconómico del país. 
Leonardi, Elías y Tortul: Sitios de patrimonio mundial como determinantes de la demanda...

En futuras investigaciones se podría entonces intentar avanzar en el estudio de los países de ingresos medio bajo y bajos del mundo en general, es decir, ampliar la muestra incluyendo países de características similares de otras regiones. Finalmente, se considera de interés profundizar el estudio de la demanda de turismo internacional en ALC, estudiando el impacto diferencial de la cantidad de SPM según el grado de especialización en turismo de los países receptores.

\section{Agradecimientos}

Este artículo se realizó en el marco del proyecto de investigación "Análisis económico de la cultura y el turismo cultural”, Secretaría de Ciencia y Técnica, Universidad Nacional del Sur. Una versión previa del mismo fue presentada en la LIII Reunión Anual de la Asociación Argentina de Economía Política. Noviembre de 2018. La Plata, Argentina.

\section{Referencias}

Adie, B. \& Hall, C. (2016). Who Visits World Heritage? A comparative Analysis of Three Cultural Sites. Journal of Heritage Tourism, 12(1): $67-$ 80. https://doi.org/10.1080/1743873X.2016.1151429

Almirón, A. V., Bertoncello, R., Kuper, D., \& Ramírez, L. (2008). El turismo como impulsor del desarrollo en Argentina. Una revisión de los estudios sobre la temática. Aportes y transferencias, 12(1), 57-86. http://nulan.md p.edu.ar/363/

Ashworth, G., \& Turnbridge, J. (2000). The tourist-historic city. Retrospect and Prospect of Managing the Heritage City. Routledge.

Ashworth, G. \& Van Der Aa, B. (2002). Bamyan: Whose Heritage Was It and What Should We Do About It? Current Issues in Tourism, 5(5), 447-457. https://doi.org/10.1080/13683500208667934

Banco Mundial. (s. f.). World Bank Country and Lending Groups. Consultado el 12 de agosto de 2019. https://datahelpdesk.worldbank.org/kno wledgebase/articles/906519 
Banco Mundial. (s. f. a). Indicadores del Desarrollo Mundial. Consultado el 17 de julio de 2019. https://datos.bancomundial.org/indicador

Breakey, N. (2012). Study in of World Heritage Visitors: The Case of The Remote Riversleigh Fossil Site. Visitor Studies, 15 (1): 82-97. https: //doi.org/10.1080/10645578.2012.660845

Brida, J. G., Lanzilotta, B. \& Risso, W. A. (2011). Turismo y crecimiento económico: el caso de Uruguay. PASOS Revista de turismo y patrimonio cultural, 6(3), 481-492. https://doi.org/10.25145/j.pasos.2008.06.036

Brida, J. G., Pereyra, J. S., Pulina, M. \& Such Devesa, M. J. (2013). Causalidad entre turismo y crecimiento económico de largo plazo: una revisión crítica de la literatura econométrica. Innovar, 23(47), 53-64. https://revistas.unal.edu.co/index.php/innovar/article/view/40245

Brida, J. G., Pereyra, J. S., Devesa, M. J. S. \& Aguirre, S. Z. (2008). La contribución del turismo al crecimiento económico. Cuadernos de Turismo, (22), 35-46. https://revistas.um.es/turismo/article/view/4793 1

Buckley, R. (2004). The Effects of World Heritage Listing on Tourism to Australian National Parks. Journal of Sustainable Tourism, 12(1): 70-84. https://doi.org/10.1080/09669580408667225

Comité Intergubernamental de protección del Patrimonio Mundial cultural y natural. (2005). Directrices Prácticas para la aplicación de la Convención del Patrimonio Mundial. Centro del Patrimonio Mundial de la UNESCO. https://whc.unesco.org/archive/opguide05-es.pdf

Cuadrado Roura, J. R., \& López Morales, J. M. (2015). El turismo, motor del crecimiento y de la recuperación de la economía española [documento de trabajo No. 4, Instituto Universitario de Análisis Económico y Social, Universidad de Alcalá]. http://hdl.handle.net/10017/21517

Drost, A. (1996). Developing Sustainable Tourism for World Heritage Sites. Annals of Tourism Research, 23(2), 479-484. https://doi.org/10.1016/ 0160-7383(96)83345-7 
Leonardi, Elías y Tortul: Sitios de patrimonio mundial como determinantes de la demanda...

Galvis, L. A., \& Aguilera, M. M. (2010). Determinantes de la demanda por turismo hacia Cartagena, 1987-1998. Lecturas de Economía, 51(51), 4787. https://doi.org/10.17533/udea.le.n51a4869

Hardiman, N., \& Burgin, S. (2013). World Heritage Area Listing of the Greater Blue Mountains Did It Make a Difference to Visitation? Tourism Management Perspectives, 6, 63-64. https://doi.org/10.101 6/j.tmp.2012.12.002

Herbert, D. (2001). Literary Places, Tourism and the Heritage Experience. Annals of tourism research, 28(2), 312-333. https://doi.org/10.1016/S0 160-7383(00)00048-7

Kayahan, B., \& Vanblarcom, B. (2012). Cost benefit analysis of UNESCO World Heritage Site Designation in Nova Scotia. Review of Economic Analysis, 4(2), 247-273. https://openjournals.uwaterloo.ca/index.php/ rofea/article/view/1386

Leonardi, V., Elías S., \& Tortul M. (2018). Sitios patrimonio de la humanidad y turismo: una aproximación a su impacto en América Latina y Caribe [ponencia]. LIII Reunión Anual Asociación Argentina de Economía Política, 14, 15 y 16 de noviembre. https://aaep.org.ar/anales/wor ks/works2018/leonardi.pdf

Lim, C. (2006). A Survey of Tourism Demand Modeling Practice: Issues and Implications. En L. Dwyer \& P. Forsyth (eds), International Handbook on the Economics of Tourism (pp. 45-72). Edward Elgar Publising.

Nguyen, T. \& Cheung, C. (2014). The Classification of Heritage Tourists: A Case of Hue City, Vietnam. Journal of Heritage Tourism, 9(1): 35-50. https://doi.org/10.1080/1743873X.2013.818677

Organización Mundial del Turismo (2019). Barómetro OMT del Turismo Mundial y anexo estadístico 2019, 17(1). https://doi.org/10.18111 /wtobarometeresp.2019.17.1.1

Palau-Saumell, R., Forgas-Coll, S., Sánchez-García, J. \& Prats-Planagumà, L. (2013) Tourist Behavior Intentions and The Moderator Effect of 
Knowledge of UNESCO World Heritage Sites: The case of La Sagrada Familia. Journal of Travel Research, 52(3): 364-376. https://doi.org/10 $.1177 / 0047287512465959$

Patuelli, R., Mussoni, M., \& Candela, G. (2013). The Effects of World Heritage Sites on Domestic Tourism: A Spatial Interaction Model for Italy. Journal of Geographical Systems, 15(3), 369-402. http://dx.doi.org /10.6092/unibo/amsacta/4180

Pocock, D. (1997) Some Reflections on World Heritage. Area 29(3): 260268. https://doi.org/10.1111/j.1475-4762.1997.tb00028.x

Porto, N., Rucci, A. C., \& Ciaschi, M. (2017). Especialización turística y accesibilidad en sitios patrimoniales del Mercosur. TRANSITARE. Revista de Turismo, Economía y Negocios, 3(1), 1-31. http://sedici.u nlp.edu.ar/handle/10915/78628

Pricewaterhousecoopers LLP. (2007). The Costs and Benefits of UK World Heritage Status in the UK. Department for Culture, Media and Sport. http://www.culture.gov.uk/images/publications/PwC_casestudies.pdf

Rodríguez Brindis, M. A. (2015). La contribución del turismo al crecimiento económico de México: Un análisis por ramas características del sector. Nova Scientia, 7(13), 337-351. http://www.scielo.org.mx/scielo.php?s cript=sci_arttext\&pid=S2007-07052015000100018\&lng=es\&tlng=es

Roh, T. S., Bak, S., \& Min, C. K. (2015). Do UNESCO Heritages Attract More Tourists? World Journal of management, 6(1), 123-200. http: //dx.doi.org/10.21102/wjm.2015.03.61.15

Ruiz Lanuza, A., \& Pulido Fernández, J. I. (2015). El impacto del turismo en los Sitios Patrimonio de la Humanidad. Una revisión de las publicaciones científicas de la base de datos Scopus. PASOS Revista de Turismo y Patrimonio Cultural, 13(5), 1247-1264. https://doi.org/10.2 5145/j.pasos.2015.13.084

Shackley, M. (1998). Visitor Management: Case Studies from World Heritage Sites. Butterworth-Heinemann. 
Leonardi, Elías y Tortul: Sitios de patrimonio mundial como determinantes de la demanda...

Schenkel, E., \& Pinassi, A. (2015). Patrimonios de la Humanidad: estrategia de la política turística en la Argentina. Revista Universitaria de Geografía, 24(2): 41-67. https://www.redalyc.org/articulo.oa?id=383243530002

Song, H., Li, G., Witt, s. f., \& Fei, B. (2010). Tourism Demand Modelling and Forecasting: How Should Demand Be Measured? Tourism Economics, 16(1), 63-81. https://doi.org/10.5367/000000 010790872213

Su, Y., \& Lin, H. (2014). Analysis of International Tourist Arrivals Worldwide: The role of World Heritage Sites. Tourism management, (40), 46-58. https://doi.org/10.1016/j.tourman.2013.04.005

Tisdell, C., \& Wilson, C. (2001). World Heritage Listing of Australian Natural Sites: Tourism Stimulus and Its Economic Value. Economic Analysis and Policy, 32(2), 27-49. https://doi.org/10.1016/S03135926(02)50017-5

Tucker, H., \& Carnegie, E. (2014) World Heritage and the Contradictions of 'Universal Value'. Annals of Tourism Research, 47, 63-76. https: //doi.org/10.1016/j.annals.2014.04.003

Tucker, H., \& Emge, A. (2010). Managing a World Heritage Site: The Case of Cappadocia, Anatolia. An International Journal of Tourism and Hospitality Research, 21(1), 41-54. https://doi.org/10.1080/13032917 .2010 .9687089

Türker, N. (2013). Host Community Perceptions of Tourism Impacts: A Case Study on the World Heritage Site of Safranbolu, Turkey. Revista de Cercetare şi Intervenție Socială, (43), 115-141. https://www.ceeol.co $\mathrm{m} / \mathrm{search} /$ article-detail id $=165930$

UNESCO (s. f.) Patrimonio Mundial. Consultado el 20 de julio de 2019 https://es.unesco.org/themes/patrimonio-mundial

UNESCO (s. f. a). World Heritage List. Consultado el 20 de julio de 2019. http://whc.unesco.org/en/list/ 
UNESCO (2021). Creación de una Red de Cooperación en Patrimonio Cultural Inmaterial y Enseńanza Superior para América Latina y el Caribe. Servicio de prensa. Creación de una Red de Cooperación en Patrimonio Cultural Inmaterial y Enseńanza Superior para América Latina y el Caribe. http://www.unesco.org/new/es/media-services /single-view-tv-release/news/establishment_of_a_latin_american_and _caribbean_cooperation

Van Der Aa, B. (2010) Preserving the heritage of humanity? Obtaining World Heritage Status and the impacts of listing [tesis de doctorado, University of Groningen. https://www.rug.nl/research/portal/publications/pu b(1147a17f-53c6-4167-8b58-36390ad91b10).html 